Purdue University Purdue e-Pubs

ECE Technical Reports

Electrical and Computer Engineering

3-1-1999

\title{
Switching Characteristics of Generalized Array Multiplier Architectures and their Applications to Low Power Design
}

Khurram Muharnmad

Purdue University School of Electrical and Computer Engineering

Dinesh Somasekhar

Purdue University School of Electrical and Computer Engineering

Kaushik Roy

Purdue University School of Electrical and Computer Engineering

Follow this and additional works at: http://docs.lib.purdue.edu/ecetr

Muharnmad, Khurram; Somasekhar, Dinesh; and Roy, Kaushik, "Switching Characteristics of Generalized Array Multiplier Architectures and their Applications to Low Power Design" (1999). ECE Technical Reports. Paper 37.

http://docs.lib.purdue.edu/ecetr/37

This document has been made available through Purdue e-Pubs, a service of the Purdue University Libraries. Please contact epubs@purdue.edu for additional information. 


\title{
SWITCHING CHARACTERISTICS OF GENERALIZED ARRAY MULTIPLIER ARCHITECTURES AND THEIR APPLICATIONS TO LOW POWER DESIGN
}

\author{
KHURRAM MUHAMMAD \\ DINESH SOMASEKHAR \\ KAUSHIK ROY
}

TR-ECE 99-4

MARCH 1999

SCHOOL OF ELECTRICAL

AND COMPUTER ENGINEERING

PuRduE UNIVERSITY

WEST LAFAYETTE, INDIANA 47907-1285 


\title{
Switching Characteristics of Generalized Array Multiplier Architectures and their Applications to Low Power Design ${ }^{1}$
}

\author{
Khurram Muhammad, Dinesh Somasekhar and Kaushik Roy \\ Email:khurram@ecn.purdue.edu,somasekh@ecn.purdue.edu and kaushik@ecn.purdue.edu \\ School of Electrical and Computer Engineering, \\ Purdue University, West. Lafayette, IN 47907
}

February 22, 1999.

\begin{abstract}
This paper presents several new array multiplier architectures for reducing the switching activity in general digital signal processing applications. A general cellular structure is described which can be used to obtain any array multiplier suitable for a given application. The switching activity at the output nodes of the cells in this structure is analyzed and compared with a tree multiplier based on $4: 2$ compressors. It is shown that the relative improvement in power is a function of statistical properties of the signal. It is also shown that selection of appropriate array architecture can give up to $40 \%$ reduction in switching activity compared to a tree multiplier, and more than 3 times less switching activity compared to the widely used least-szgnzficant-bzt-first array multiplier for commonly occurring situations. We also outline applications of the proposed multipliers to the areas of low power quantization, reconfigurable computing and high-level synthesis for low power.
\end{abstract}

'This work was supported in part by DARPA (F336I5-95-C-1625), NSF CAREER award (9501869-MIP),Rockwell, AT\&T and Lucent foundation. 


\section{INTRODUCTION}

With the recent trend in increasing mobility and performance in small hand-held mobile communication and portable computing equipment, low power has become an important design factor. New features are continually provided using DSP algorithms which are dominated by three basic operations; add, shaft and multiply. Many DSP algorithms can be implemented such that the data is processed in carry save (CS) format. as this format yields zero cost of accumulation [1] in multiply-and-accumulate (MAC) operation. The conversion of the result to normal binary forrn can be delayed for as long as possible for the given algorithm sirce it results in a significantly faster implementation. Consider, for example, a digital filter implementat:on. In such an application, the intermediate result which is the accumulation of a given inner product of data and the coefficient can be kept stored in CS format, with the CS to binary conversion taking place only after the final result is computed in CS form. Consequently, multiplier architectures processing detta in CS format are of particular interest.

Multiplication operations are considered to be the dominant computation in DSP algorithms [2], [3]. Since, computation directly results in dynamic power consumption [4] it is an equally important factor when considering dynamic power dissipation of such algorithms. In general, high-performance DSP architectures as:e required in mobile units which process data at high transmission rates, or in a portable computer providing advance multimedia features. For this reason, such units are generally constructed with pipelined array multipliers. If the latency of the pipelined architecture is an important consideration, a pipelined tree multiplier can be used. Both types of multipliers can be easily pipelined using the conventional register based approach, or by using wave pipelining. Over the past few years, a number of papers have addressed multiplier topologies for a variety of applications [1], [6], [7]. In particular, array structures proposed in [6] address pipelining of recursive digital filters using most significant bit (MSB) first digit serial arithmetic. However, to the best of our knowledge, no work has been reported in literature which address dynamic switching activity trade-offs between popular multiplier architectures as a function of statistical properties of inputs.

In this paper, we esplore array structures from the point of view of dynamic power dissipation. Contrary to the expectation that any ordering of array multiplier would yield similar dynamic power dissipation performance, we will show that more than $\mathbf{3}$ times reduction in switching activity may be possible compared to the commonly used least significant bit (LSB) first array multipliers (also known as right-left multipliers), depending on the signal characteristic of input signals. This is because a salient feature of computation in DSP algorithms is that the computations are governed by the statistical properties of the underlying process generating data. In general, data signals are correlated and consequently, rapid changing data is seldom processed. Hence, we will explore the effects of signal statistics on the output switching activity in various array structures in order to assess the feasibility of using a given structure under the condition of known or predictable signal statistics. We will show that re-ordering of partial product addition can result 
in significant reduction in switching activity (hence, dynamic power) if the signal statistics are known $a$ przori. This observation leads to new array multiplier architectures which form hybrids of MSB-first and LSB-first structures. We also discuss the application of such multipliers to low power iniplementation of DSP algorithms and to the general area of reconfigurable computing.

The main objective of this work is to identify what type of architectures are best suited for processing signals with known statistical properties for reduced dynamic power dissipation? There are three major contributions: of this work:

- We propose hybrid-array structures which combine LSB-first and MSB-first types of array multipliers. For appropriate signal conditions, these structures are shown to significantly reduce dynamic power dissipation.

- The switching characteristics of array multipliers are compared with a tree multiplier based on $4: 2$ compressors as well as the most commonly used LSB-first multiplier to show the region of strength of each architecture. Hence, this work can be used to formulate an appropriate strategy for selecting the best order of partial product addition for reducing power dissipation in a given DSP task. Alternatively, when processing signals with known statistical properties, one can formulate a strategy for applying signals to the multiplier inputs in an order which most effectively reduces dynamic power dissipation.

- The architectures presented in this paper provide new insights to the general area of low power design and reconfigurable computing.

This paper is organized in to five sections. Section II describes the array multiplier architectures considered in this work. Section III presents a simulation based study of the switching characteristics of output nodes in the architectures considered. The signal models used to compute the performance of these multipliers are also explained in this section. Section IV discusses the applications of these struct ures to general signal processing algorithms. Finally, section V concludes this paper.

\section{Multiplier Architectures}

We will first present a simple approach for obtaining various types of array multipliers. Figure 1 shows a template for a cellular array structure which serves as the basis for generating different types of 8-bit array multipliers. Each location in this matrix can be occupied by a cell which can be an and gate (AND), a half adder (HA) or a full adder (FA). In the sequel, the cell at location $i, j$ will be referred to as $c_{i, j}$. As an example, the cells on four corners are shown labeled in the figure. Let $\mathbf{A}=a_{0}, a_{1}, \ldots, a_{N-1}$ and $\mathrm{B}=b_{0}, b_{1}, \ldots, b_{N-1}$ represent the input vectors applied at right and top, respectively. The output is represented by $\mathbf{P}=p_{0}, p_{1}, \ldots, p_{2 N-1}$. Then each partial product $a_{i} . b_{j}$, where $i, j=0,1, \ldots, N-1$ must be added in the appropriate relative position to obtain the correct value of $\mathbf{P}$. In figure 1 we have shown the structure of LSB-first type array multiplier by the colored cells comprising a parallelogram. In this figure, the continuous lines show presence of connections, while the dashed lines show absence of them. 
Hence, the active connections in a CS type of array multiplier are shown using the continuous lines. The connections from primary inputs to appropriate cells are not shown explicitly, and are assumed implicit to reduce clutter. By counting the number of active inputs, one can determine the type of cell. Hence, the cells in row \#O are all AND gates, whereas the seven rightmost cells in row \#1 are HAs. The cells accepting three active inputs are FAs. Note that the inputs are counted by considering tle implicit input $a_{i} \cdot b_{j}$ which is not shown. The resulting CS array multiplier structure is shown on the right in figure 1 for clarity.

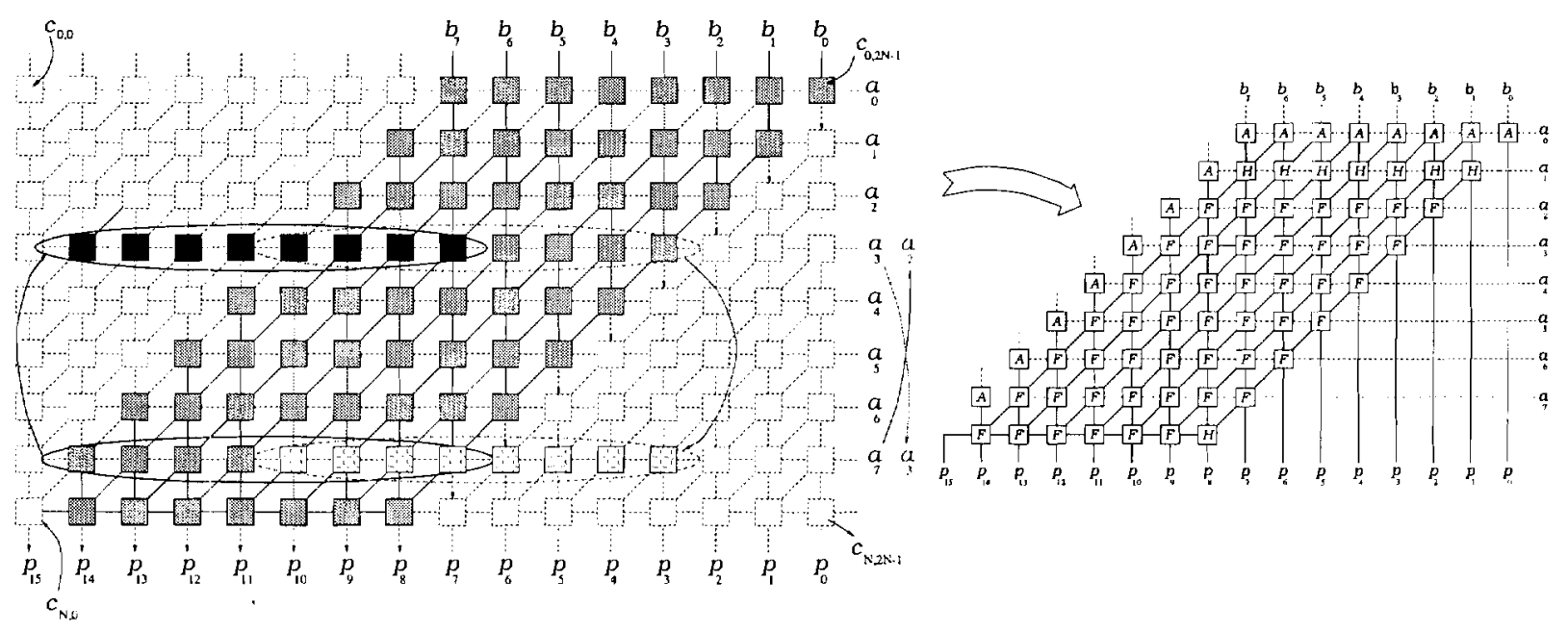

Fig. 1. Basic template for constructing array multipliers.

Now, the goal of an array multiplier is to add the partial products from cells which occupy the same column in the cellular array structure shown in figure 1. The order in which these partial products are added is not important, we only need to ensure that only the products in the same column are added (in addition to the carry's generated from the cells in the adjacent column on right). Hence, one can exchange rows \#3 and \#7 as shown in figure 1. Cells in row \#3 after moving to row \#7 are shown by cells shaded by circles. The cells in row \#7 after moving to row \#3 are shown by dark colored cells. Now, we only need to ensure that carry's generated from next rows are correctly added, which may require extra cells. Let $\mathcal{R}=\left\{r_{0}, r_{1}, r_{2}, \ldots, r_{N-1}\right\}$ be the set of indices which represents an ordering of successive additions of rows of partial products. Then, the ordering given by $r_{i}=i$ for $\mathrm{i}=0,1, \ldots, \mathrm{N}-1$ expresses the LSB-first multiplier shown in figure 1. The MSB-first multiplier can also be expressed similarly by the ordering $r=N-1-i$ for $i=0,1, \ldots, \mathrm{N}-1$. Clearly, there are $\mathrm{N}$ ! ways to construct carry save array multipliers. Each of these multipliers mays be constructed using propagation of carry in either ripple form or CS form or a combination of these. This formulation is the basis of generating various architectures of interest which are evaluated for their switching activity performance in this paper. 


\section{A. LSB-First Multipliers}

The LSB-first multiplier can be constructed either using the CS format shown in figure 1, or by using ripple carry structure. We will refer the former as LSB-first CS multiplier and the latter as the LSBfirst R P multiplier, respectively. LSB-first RP multiplier is the most well-known and widely used array structure for multiplication and is obtained from the cellular array of figure 1 by turning off the diagonal lines (by making them dashed) and turning on the horizontal dashed lines (by making them continuous) which connect cell $c_{i, j}$ to $c_{i, j+1}$ for all cells $c_{i, j}, \mathrm{i}=0,1, \ldots, \mathrm{N}-1$ and $\mathbf{j}=\mathrm{N}-i, N-i+1, \ldots, 2 N-i-2$ (right-most cell excepted) in the LSB-first CS multiplier of figure 1. The vector. merge row (row \#N+1) is no longer required. The advantage of using CS format is the reduction in propagation delay through the multiplier. LSB-first RP multiplier has 30\% longer critical path as compared to the LSB-first CS multiplier. Inı this work, we consider both since our objective is to highlight the switching characteristics of various array multipliers.

\section{B. MSB-First Multipliers}

An MSB-first multiplier place MSBs of $\mathbf{A}$ input at the top row positions as shown in figure 2. The main idea is to flip the cells in the cellular array of figure 1 along a horizontal axis such that row \# $i$ is moved to row $\#(\mathrm{~N}-1-i)$, for $i=0,1, \ldots, N-1$. This results in a MSB-first multiplier [6]. The multiplier can be constructed by propagating the carry in either CS form, or can be ripple in a fashion identical to the LSB-first RP multiplier. The multiplier using CS format has been presented in [6] for pipelining recursive digital filters. A major advantage of the MSB-first CS multiplier is that the delay through vector merge stage can be reduced by taking advantage of the fact that the MSB-first array produces the MSBs before the LSBs. Hence, a carry-select structure can be constructed in the region occupied by cells $c_{i, j}$ for $i>\mathbf{j}$ to improve the vector merge delay. Consequently, MSB-first CS array multiplier can improve the speed of multiplication [6]. The observation that MSBs of product are available before the LSBs is fundamental to the construction of the MSB-first RP multiplier shown in figure 2(b). In contrast to a LSB-first RP multiplier, it has the same propagation delay as the LSR-first CS multiplier and offers an attractive alternative to it.

\section{Harid Multipliers}

A hybrid multiplier is obtained by any ordering of elements of $\mathcal{R}$ which is not monotone. Note that there is only one monotonically increasing ordering of the elements of $\mathcal{R}$ and it leads to the LSB first structure. Similarly, the only monotonically decreasing ordering leads to the MSB first structure. Any ordering other than these two leads to a hybrid array multiplier. In this paper, we consider only two types for hybrid structures. The first structure places $L$ consecutive LSB bits of operand $A$ as $L$ top most rows. This structure is shown on left in figure 3. The second structure places $L$ consecutive MSB bits of operand $A$ as $L$ top most rows and is shown on right in figure 3 . We will refer to the former as 


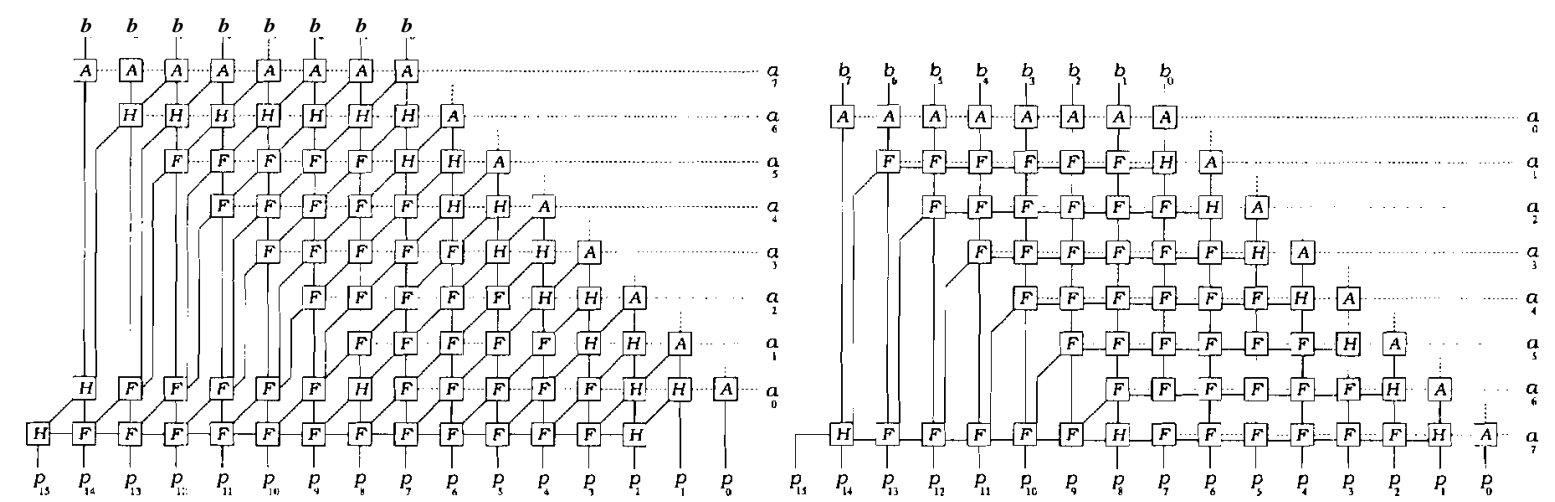

Fig. 2. Structures for MSB-first multipliers; (left) MSB-first CS multiplier, and, (right) MSB-first RP multiplier.

hybrid LSB-first multiplier and the latter as hybrid MSB-first multiplier, respectively. Botli of these can be constructed either by using ripple carry or by using CS format. Hence, there are four ways to implement a hybrid multiplier which puts $L$ top most rows of one type of multiplier above the other (i.e. LSB-first over MSB-first or vice versa). The multiplier on left in figure 3 puts $\mathrm{L}=\mathbf{3}$ top rows of the LSB-first CS multiplier over $N-L=5$ top rows of the MSB-first CS multiplier. We will refer to such a multiplier as hybrid LSB-first CS/CS multiplier with $L=\mathbf{3}$. Similarly, the multiplier on right in 3 puts $L=\mathbf{3}$ top most rows of MSB-first RP multiplier over $N-L$ top most rows of LSB-first CS multiplier. This multiplier will be referred to as hybrid MSB-first RP/CS multiplier with $L=3$. We can obtain three more types of $L=\mathbf{3}$ hybrid multipliers for each of these cases by considering the remaining three combinations of adding carrys in the two parts of the multiplier.

Each type of hybrid multiplier implementation requires a different overhead and has a different length of critical path. Since the goal of this work is to develop an understanding of the switching trade-offs in various multipliers, we will only consider implementations which place $L$ consecutive rows of one type of multiplier over the other. The reason for focusing on such architectures is because DSP applications, in general, process data streams whose properties can only be predicted or controlled over a part of the word-length. For example, if the signal strength reduces, consecutive MSBs of the data-stream become zeros (assuming a sign-magnitude representation). Similarly, "less important" data values may be further quantized by truncating some LSBs, thereby resulting in the data-stream having zeros at the corresponding locations. It will be shown that the proposed hybrid multipliers yield substantial improvement in switching activity reduction compared to a tree multiplier (constructed using $4: 2$ compressors) as well as the simple LSB-first or MSB-first multipliers under appropriate signal conditions. The multiplier structure shown on left in figure $\mathbf{3}$ is entirely CS structure, and its speed can be increased by using a carry select structure similar to the one proposed in [6]. The multiplier on right in 3 has the same delay as a LSB-first CS array multipl:er despite the fact that the MSB-first part ripples the carry. The reason for considering this structure is that it requires a smaller overhead cells required to ensure that all partial product sums and 
carrys are added at appropriate locations.
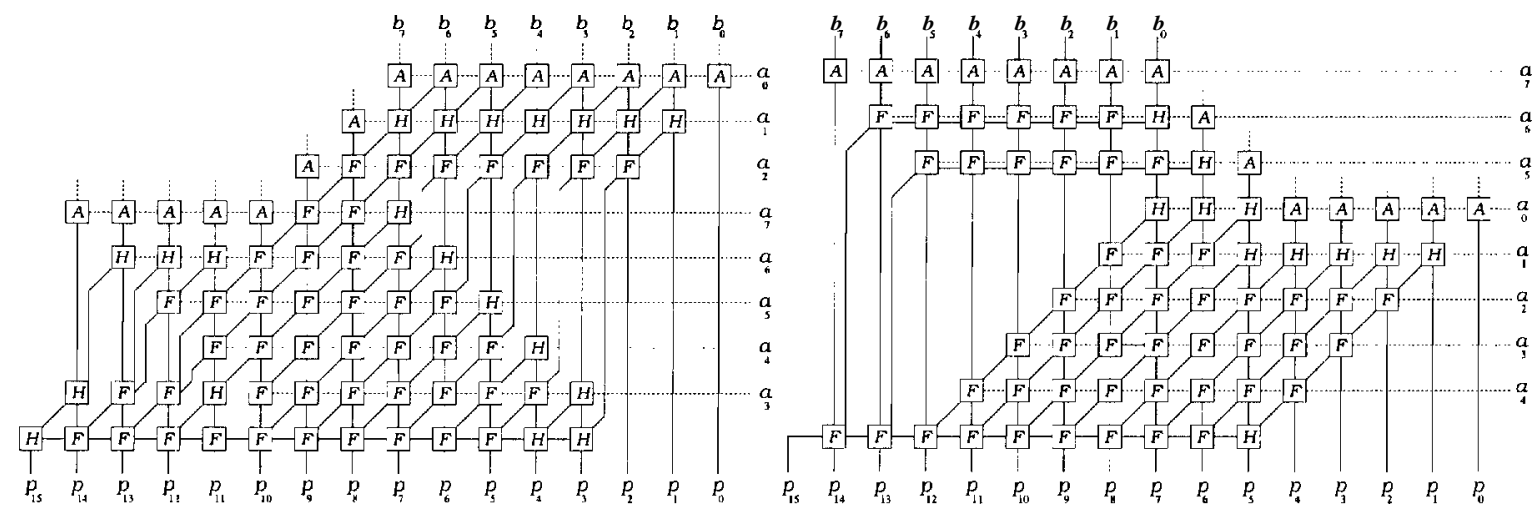

Fig. 3. Structures for hybrid multipliers; (left) hybrid LSB-first CS/CS multiplier, and, (right) hybrid MSB-first RP/CS multiplier.

\section{Switching Characteristics of Multipliers}

We first investigate the switching characteristics of the multipliers presented in the previous section qualitatively. Let us first consider the LSB-first multipliers. A close observation of the multiplier in figure 1 shows that if successive inputs are applied such that their LSBs are zeros in operand A, the corresponding top rows of the multiplier will be turned off as the evaluated partial products would all be zeros. Ary input which has $a_{0}=1$ will place the vector $\mathrm{B}$ at the output of the first row of partial product outputs. These values will propagate downwards even if the next LSBs in A are all zeros. Hence, switching activity can only be reduced if successive inputs applied at the input $\mathrm{A}$ ensure that when a bit $a_{j}$ is 1 , all $a_{i}$ 's are zeros for $i<j$. Similarly, we notice that if the successive inputs applied at the B inputs are such that M MSB bits are zeros, then the cells $a_{i, j}$ such that $j=\mathrm{i}+\mathrm{k}$ for $\mathrm{k}:=0,1, \ldots, \mathrm{L}-1$ along the diagonal (columns of partial product generators) in the cellular array are all turned off. Hence, no sum or carry output transitions in these cells. Hence, low over-all switching activity can be ensured if the inputs applied to this multiplier are ordered to ensure that they cause smaller switching activity.

Similar observations are made for the MSB-first and hybrid multipliers. The "best" input conditions for these multipliers are summarized in table I and can be verified by a careful study of figures $1-\mathbf{3}$. Next, in order to obtain a quantitative behavior of these multipliers, we will use two signal models which are described next.

\section{A. Signal Models}

In the first model we only vary the signal strength to determine the switching characteristics. Hence, successive samples of signals are assumed to be uncorrelated and drawn from a uniform distribution. It has been shown in [10] that the switching activity in the LSB-first RP multiplier primarily depends on the input signal strength. Hence, we apply all possible combinations of fixed signal strengths in an $N$-bit 


\begin{tabular}{|c|c|c|c|c|}
\hline \multirow{2}{*}{$\begin{array}{c}\text { Multiplier } \\
\text { Type }\end{array}$} & \multicolumn{2}{|c|}{ Favorable Conditions } & \multicolumn{2}{|c|}{ Overhea ds } \\
\hline & at Input $\mathbf{A}$ & at Input B & Vector Merge & Wifing \\
\hline LSB-first CS & LSBs zeros & MSBs zeros & $\mathrm{N}-1$ cells & None \\
\hline LSB-first RP & LSBs zeros & MSBs zeros & Not Required & None \\
\hline MSB-first CS & MSBs zeros & MSBs zeros & $3(N-1)$ cells & Yes \\
\hline MSB-first RP & MSBs zeros & MSBs zeros & $N-1$ cells & None \\
\hline Hybrid LSB-first CS/CS & MSBs \& LSBs zeros & MSBs zeros & $3 N-2 L-1$ & Yes \\
\hline Hybrid MSB-first RP/CS & MSBs \& LSBs zeros & MSBs zeros & $L-1$ cells & None \\
\hline
\end{tabular}

Signal conditions Causing Low SWitching activity and overheads in the multipliers PRESENIED. Hybrid MULTIPLIERS ASSUME THAT $L$ ROWS ARE MOVED TO TOP.

multiplier by sweeping the space of possible signal strengths at the two inputs. We obtain data for these points by generating samples comprising of $i$-bits from a uniform distribution, where $i$ is varied from 1 to $\mathrm{N}$. The $\mathrm{N} \times \mathrm{N}$ possible combinations of siginal strengths of the two operands are obtained by applying signal of strength $\mathrm{i}$-bits as operand $\mathbf{A}$ and $j$-bits as operand $\mathbf{B}$, where $\mathbf{i}, \mathbf{j}=1,2, \ldots, \mathrm{N}$. This model will be referred to as the $\mathcal{U}$ model and it can be used to assess the merits of using the presented multipliers for signals whick can be represented by $\mathrm{N}$ or less bits and/or which can be re-quantized by discarding some LSBs without significantly degrading the system performance.

The second model generates correlated signals from a zero mean Gaussian distribution. These samples are represented using sign-magnitude (SM) number representation and only the magnitude of the number is applied at the inputs of the multiplier. The signal correlation in operand $\mathbf{A}$ is represented by $\rho_{A}$ and the correlation in $\mathrm{B}$ is represented by $\rho_{B}$. Four situations arise by considering all possible combinations of high and low correlations in the signals at the two inputs. The high correlation value is considered to be 0.95 , and low correlation equal to 0 . This model will be referred to as the $\mathcal{G}$ model.

\section{B. Numerical Results On Power Dissipation}

We now turn our attention to the switching activity performance of the presented multipliers. The switching activity of each multiplier was evaluated by counting the number of switches at each output of every module in the multiplier. Let $S_{c}$ denote the switching count of cell c. Then the possible cells in a multiplier are an $A N D$ gate, a HA, a FA and a $4: 2$ compressor (the $4: 2$ compressor appears in the tree multiplier). The corresponding switching metric which expresses the switch counts in these cells will be represented by $S_{A N D}, S_{H A}, S_{H A}$ and $S_{4: 2}$, respectively. The total switching metric was obtained using the following; weighting; 2 for $S_{A N D}, 3$ for $S_{H A}, S_{F A}$ and $S_{4: 2}$ (weight reflects output load capacitance 
driven by the gate output). These relative weighting factors were obtained by considering the pin loading of a typical rnodule in the array configuration. In addition, the switches at the input pins were counted separately for the given simulation and multiplied by $\mathrm{N}$ to account for input buffer drivers. The total switch counts at all outputs (including input pins), weighted by the corresponding factor were summed to obtain the switching metric for the multiplier. These weightings yield a metric which expresses the total switched capacitance in the multiplier for the given input conditions.

A similar metric was obtained for the tree multiplier by using using the same input signals. We will let $\mathcal{S}_{\text {Array }}$ and $\mathcal{S}_{\text {Tree }}$ denote the switching metrics for the array and tree multipliers, respectively, for the given input signal conditions. Then the relative advantage of using the array multiplier is defined as

$$
\eta_{\text {Tree }}=\frac{\mathcal{S}_{\text {Tree }}-\mathcal{S}_{\text {Array }}}{\mathcal{S}_{\text {Array }}} \times 100
$$

The above quantity is expressed as a percentage and shows the advantage of using the array multiplier over a tree for the given signal condition. We will refer to this quantity as percentage switching reduction. The rationale behind this normalization is to clearly indicate the relative performance of each type of array multiplier with respect to the tree structure and to quantify percentage reduction in switching activity for given signal condition. Similar quantity can be obtained for comparing the relative performance of any two multipliers. Figure 4 shows one such metric computed using the LSB-first CS multiplier as the reference for normalization. The figure shows the relative advantage of using the indicated hybrid multipliers ill comparison to the LSB-first CS multiplier by using $\mathcal{S}_{L S B-F i r s t C S}$ in place of $\mathcal{S}_{\text {Tree }}$ in equation 1. This quantity will be represented by $\eta_{\text {Array }}$, as we set the LSB-first CS multiplier as the base-line for comparison in array multipliers. It is noted that switching reduction of up to $200 \%$ (3X smaller) is possible when using a hybrid multiplier in comparison to the LSB-first CS multiplier, under appropriate signal conditions.

The result:; presented in this section were obtained by using 1000 randomly generated vectors using the $\mathcal{U}$ model. These results give rise to a surface as a function of the number of bits in the applied inputs. This surface is best shown by slicing it into different regions and showing every slice individually as in figure 4. An even better representation is to place each slice along-side as a bar chart as shown in the remaining figures. The abscissa in these figures show the number of bits in the samples (drawn from a uniform distribution) applied at the $\mathbf{A}$ input. The data samples were applied at the multiplier inputs by aligning their LSB with the zeroth indexed row/column. Hence, the successive simulations computed the switching metrics for inputs with increasing widths until metrics for all the grid points of the switching metric surface were computed. The metrics were normalized to obtain relative switching ireduction shown in the figures. The bars in each figure are composed of $\mathrm{N}$ groups. The position of a group corresponds to the number of bits in the samples applied at $\mathbf{A}$. Each group, in turn, is composed of $\mathrm{N}$ bars. The position of a bar inside a group indicates the number of bits in the samples applied at the B input. Hence, as we scan a figure from left towards right, the strength of the input signal at B input repeatedly increases and 

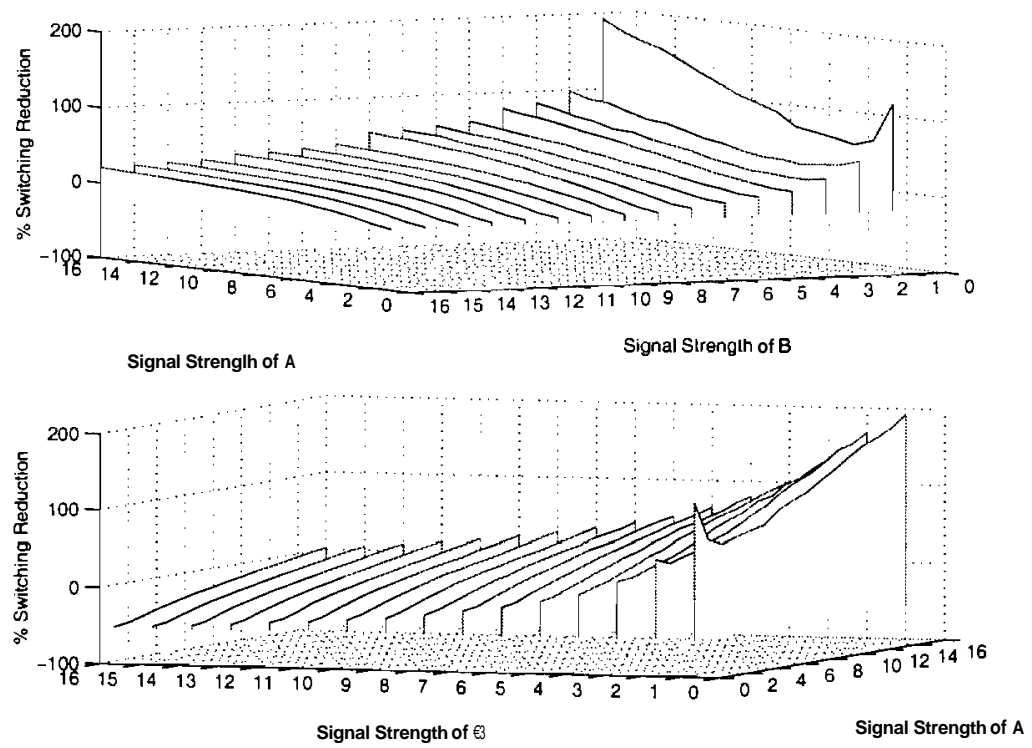

Fig. 4. $\eta_{\text {Arra:y }}$ for 16-bit hybrid array multipliers. Figure above: shows the percentage switching reduction for Hybrid LSB-first CS/CS with L=1, and, figure below: shows this surface for Hybrid MSB-First RP/CS multiplier with L=1 (normalization is performed with respect to LSB-first CS multiplier).

falls, while the strength of the signal applied at $\mathbf{A}$ continually increases.

\section{B.1 Results Using the $\mathcal{U}$ Signal Model}

Figures 5 -- 7 show $\eta_{\text {Tree }}$ as a function of signal strength in the LSB-first and MSB-first multipliers for $\mathrm{N}=8,15$ and 32 , respectively. We observe a consistent trend of the relative performance for each of these multipliers. Each of these multipliers gives gains in switching reduction for different operating conditions. As pointed out in table I, LSB-first multipliers would give an improvement when the LSBs of A input, or MSBs of B input are zeros. The first situation does not arise with this signal model, because it would require MSBs to be $1 \mathrm{~s}$ and LSBs to be $0 \mathrm{~s}$. Such a signal can only be generated by quantizing (rounding/truncating) the LSBs. However, the second condition is more realistic and we note that up to $25 \%$ reduction in switching activity is possible over tree multiplier when the signal strength of $\mathbf{A}$ is high, and B is small as they result in left-most columns of multipliers turning off. Despite the overhead of vector merge state, the CS multiplier out-performs the R P multiplier as evident by a close inspection of these figures. The MSB-first multiplier shows the gains in switching activity reduction when the signal strength at the $\mathbf{A}$ input is low. Hence, the top most rows do not switch. Larger gains are observed when the signal strength at the B input is large. The R P type multiplier clearly outperforms the CS multiplier because of smaller overhead cells. Further, the relative gains under favorable signal conditions are higher as compared to the LSB-first multipliers. Finally, both favorable situations appear at the inputs in the $\mathcal{U}$ signal model because the MSB-first multipliers reduce switching when the MSBs of both inputs are 0s (a situation which frequently arises in DSP applications). It is seen that close to $40 \%$ reduction in switching 

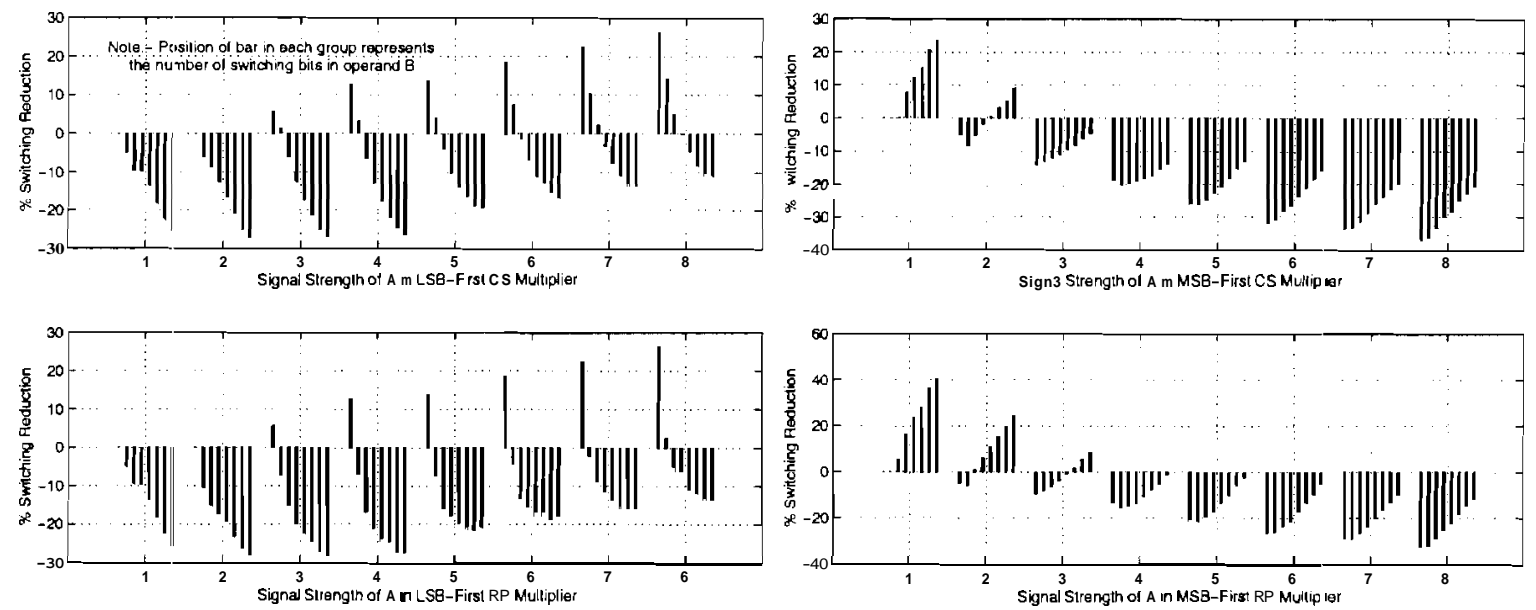

Fig. 5. $\eta_{\text {Tree }}$ for (left) 8-bit LSB-first array multiplier, and, (right) 8-bit MSB-first array multiplier as a function of the signal strength in the operands.
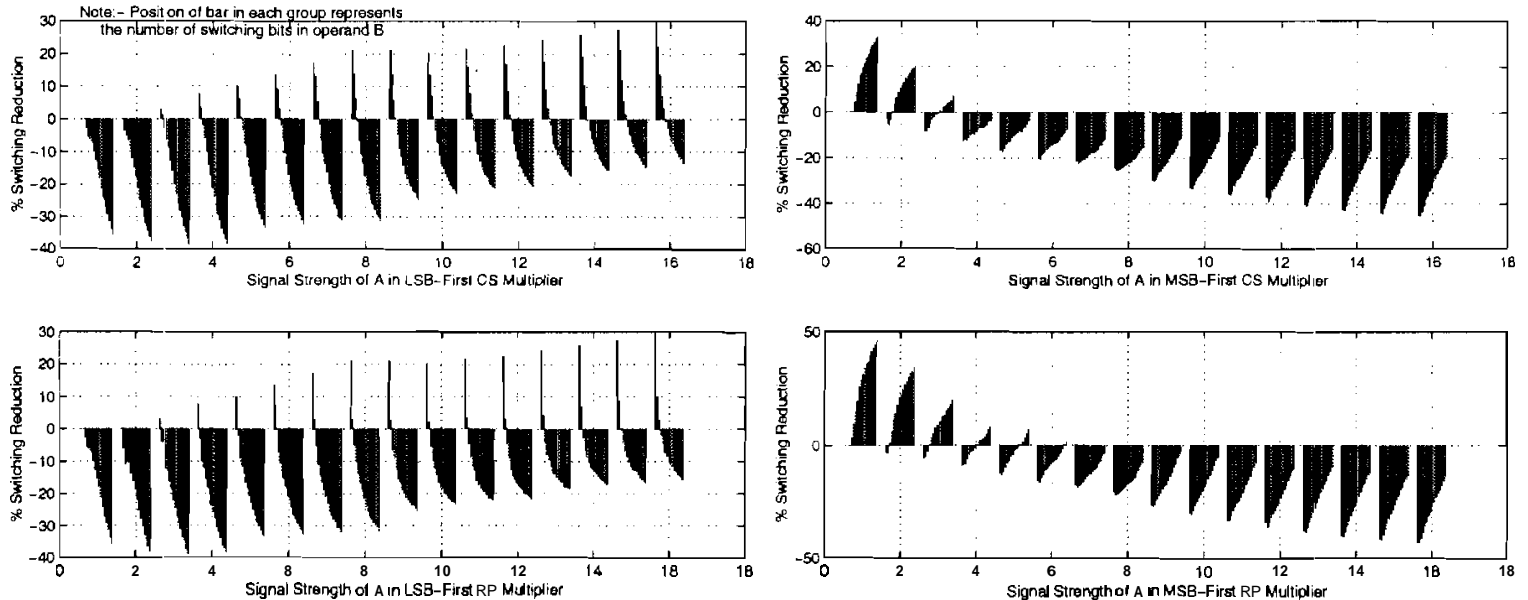

Fig. 6. $\eta_{\text {Tree }}$ for (left) 16-bit LSB-first array multiplier, and, (rightj 16-bit MSB-first array multiplier as a function of the signal strength in the operands.

activity is possible in the MSB-first RP multiplier when the signal strength of $\mathbf{A}$ is very small and B is very strong. The savings are consistent across 8, 16 and $\mathbf{3 2}$ bit multipliers.

We can also compare the relative performance of MSB-first and LSB-first multipliers. Figure 4 shown earlier indicates that LSB-first multiplier out-performs the MSB-first multiplier by up to $30 \%$ when signal strengths at both $\mathbf{A}$ and $\mathbf{B}$ inputs are very small. However, MSB-first multiplier out-performs LSBfirst multiplier for most situations giving larger relative advantage in switching reduction. Note that these results favor MSB-first type multiplier from switching activity point of view for most common signal conditions. One may notice that many multipliers used in DSP do not need all $2 \mathrm{~N}$ product bits (especially in floating point units) and MSB-first multiplier is an attractive choice since by construction it also furnishes the MSB part of the product very quickly. 

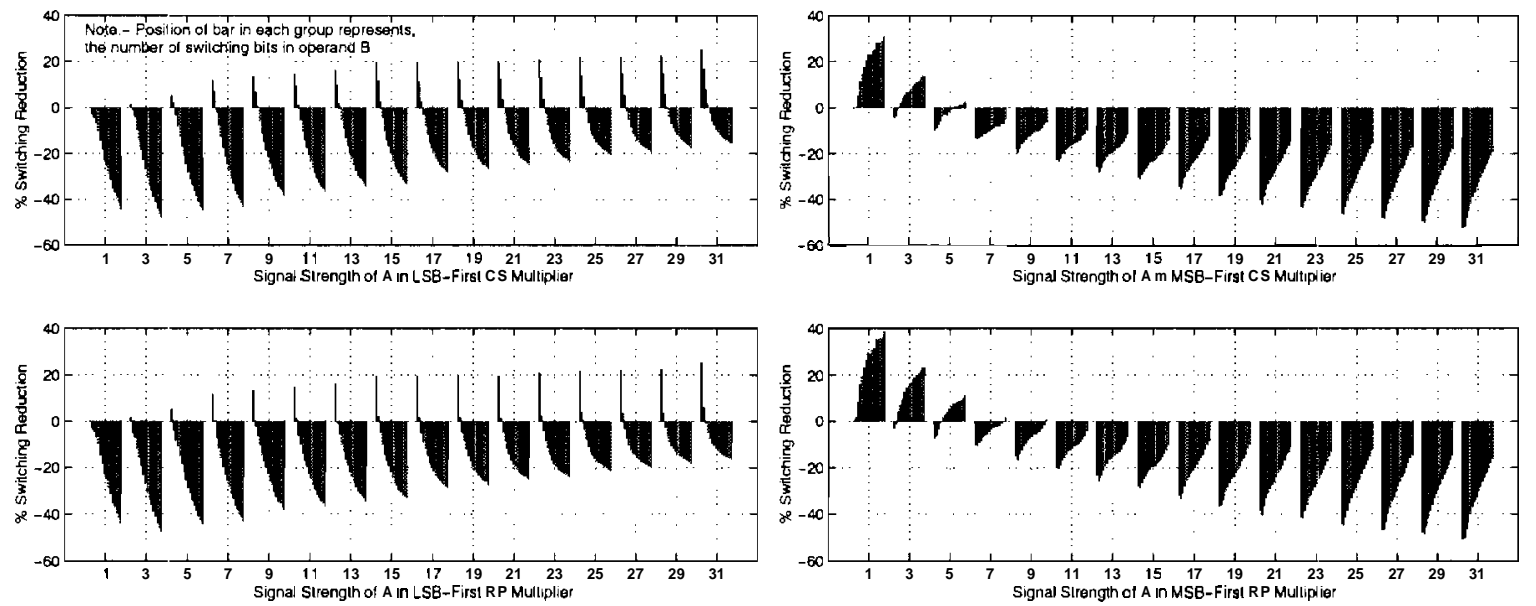

Fig. 7. $\eta_{\text {Tree }}$ for (left) 32-bit LSB-first array multiplier, and, (right) 32-bit MSB-first array multiplier as a function of the signal strength in the operands.

We next consider hybrid multipliers. The main objective of employing the hybrid multipliers presented in this paper is to take advantage of a signal whose L LSB bits are zeros. Such signal values may arise in many ways in typical DSP applications. As an example, computations may be organized as floating point type of operations in which the normalized mantissa of operands is multiplied using an array multiplier and values are expressed by using only a few MSB bits in the mantissa, depending on the accuracy required (variable preczszon arzthmetzc). Example of such a system is a digital filter implementation employing scaled coefficients for reducing performance degradation due to coefficient quantization [3]. Another example of truncation of signal's L LSB bits is a situation where the resulting degradation in accuracy can be tolerated for the application at hand. Again, examples of such a system is an FIR filters whose objective is to meet given filter specifications, however, the implementation is nnade by using a multiplier which is bigger than the least number of bits required to meet these specifications [8], [9]. This situation car easily arise in general DSP implementations where shared multipliers are used for more than one applications and resources are not exclusively dedicated to only one task. For these reasons the switching performance of the hybrid multipliers was computed by truncating L LSB bits of the signal and setting them to zeros. If L LSB bits are not set to zeros, the hybrid multiplier's switching performance will lie between that of LSB-first and MSB-first multipliers.

Next, we analyze the results shown in figures $8-10$. These figures show $\eta_{\text {Tree }}$ for 8,16 and 32-bit multipliers, respectively. The figures on left show the results for multipliers with $L=1$, and the figures on right show the results obtained for multipliers with $\mathrm{L}=2$. It is seen that hybrid MSB-first and hybrid LSB-first multipliers show improvement in performance for different signal conditions. The former shows most improvsment when the signal strength is small for $\mathbf{A}$ and large for $\mathbf{B}$. The latter shows most gains when the converse is true. The reduction in switching activity is more pronounced in the Hybrid LSB-first multiplier despite the overhead of cells required to ensure correct operation. This is due to the fact that 

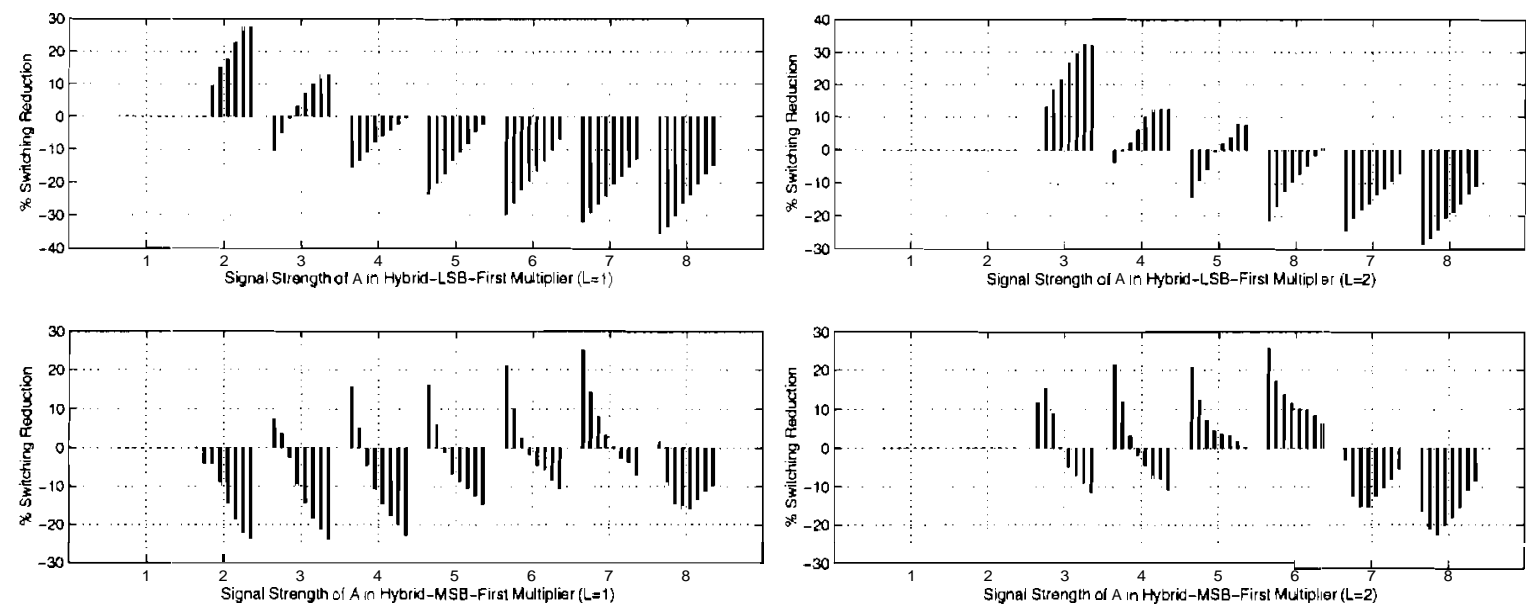

Fig. 8. $\eta_{\text {Tree }}$ for 8-bit hybrid array multipliers as a function of the signal strength in the operands. (left: $)$ L=1, and, (right) $\mathrm{L}=2$.
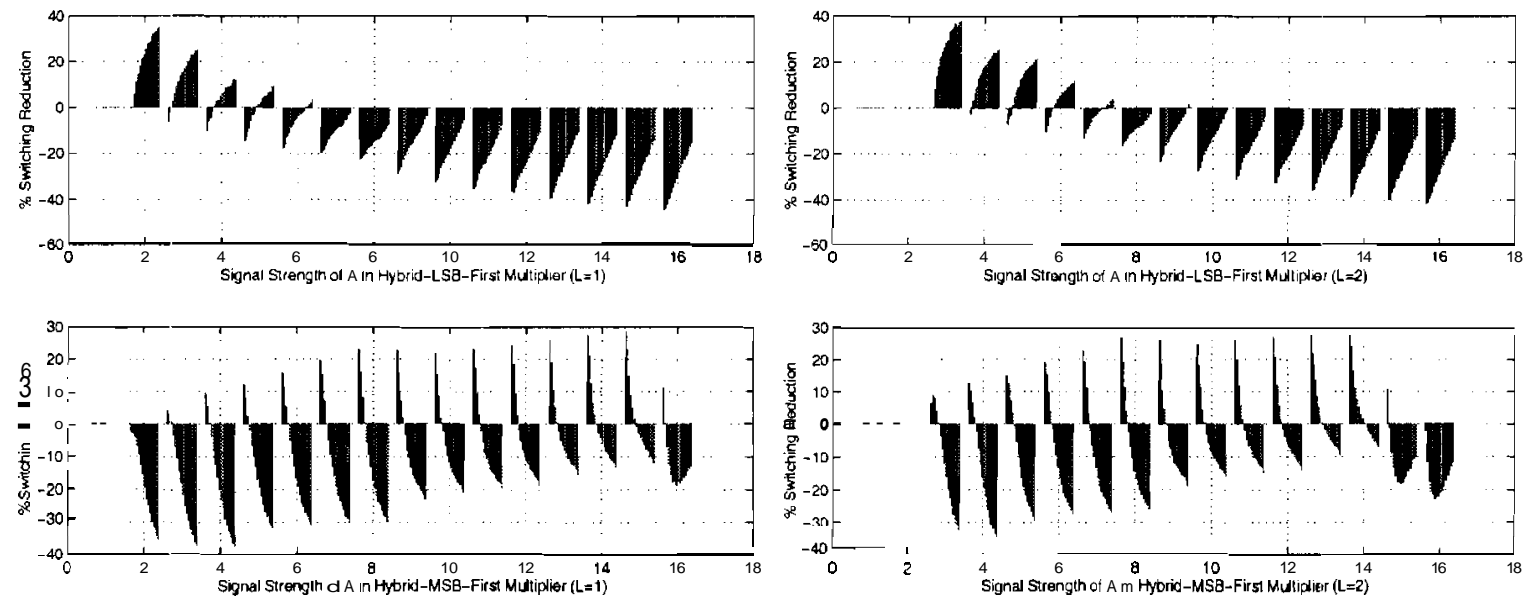

Fig. 9. $\eta_{\text {Tree }}$ for 16-bit hybrid array multipliersas a function of the signal strength in the operands. (left) $\mathrm{L}=1$, and, $($ right) $\mathrm{L}=2$.

the L LSB bit truncated signals obtained through the $\mathcal{U}$ model are more effective in turning off larger part of the multiplier since LSB-first part of the multiplier precedes the MSB-first in the former case. Significant reduction in switching activity is achieved in both cases. Further, the trends are consistent for all sizes of multiplier.

Figures 11 shows $\eta_{\text {Tree }}$ for 8 and 16-bit multipliers, respectively, with L = 3. Figure 12 shows $\eta_{\text {Tree }}$ for 16 and 32-bit multipliers, respectively, for hybrid multipliers with $\mathrm{L}=4$. The missing bars indicate that the $\mathbf{A}$ operand under the indicated signal conditions were zeros (small power, large truncation). Hence, no operations are necessary. However, the region of switching reduction moves to the the mid-region of A signal power. The relative switching activity reduction becomes larger as $\mathrm{L}$ increases The trends are consistent for all hybrid LSB-first CS/CS and hybrid MSB-first RP/CS multipliers for all sizes and values 

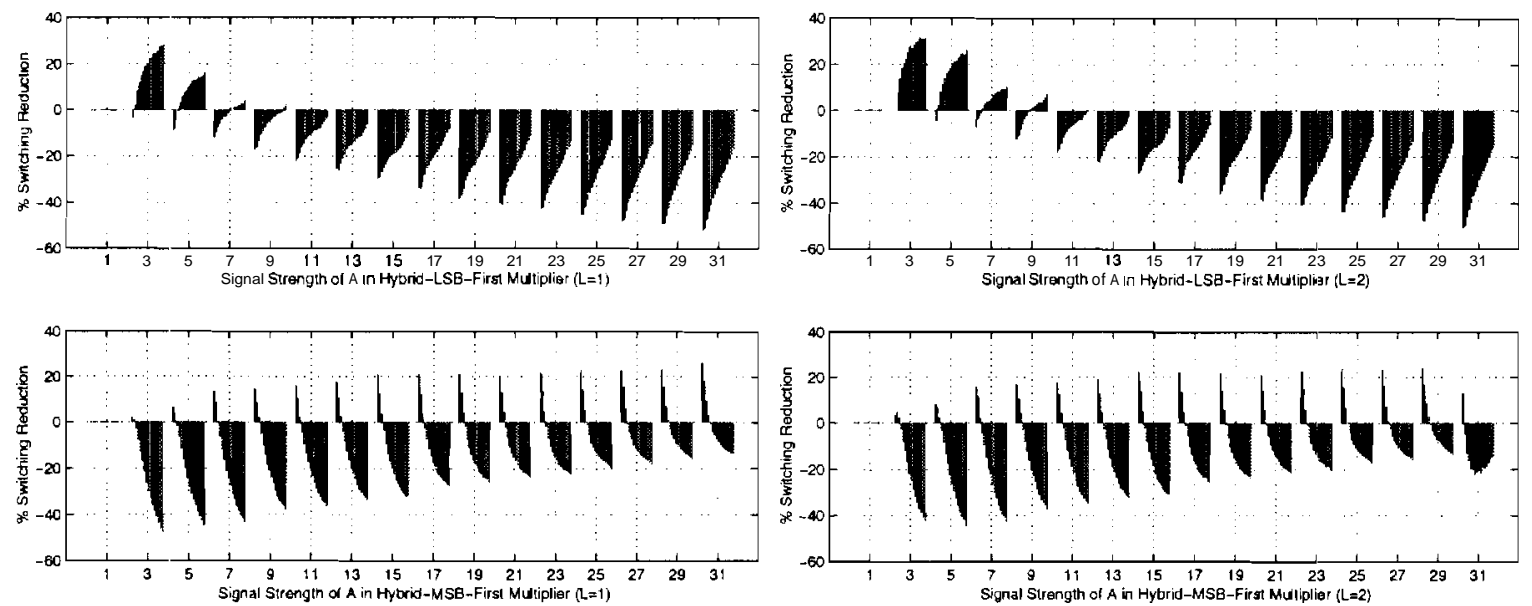

Fig. 10. $\quad \eta_{\text {Tree }}$ for 32-bit hybrid array multipliers as a function of the signal strength in the operands. (left) L=1, and, (right) $\mathrm{L}=2$.
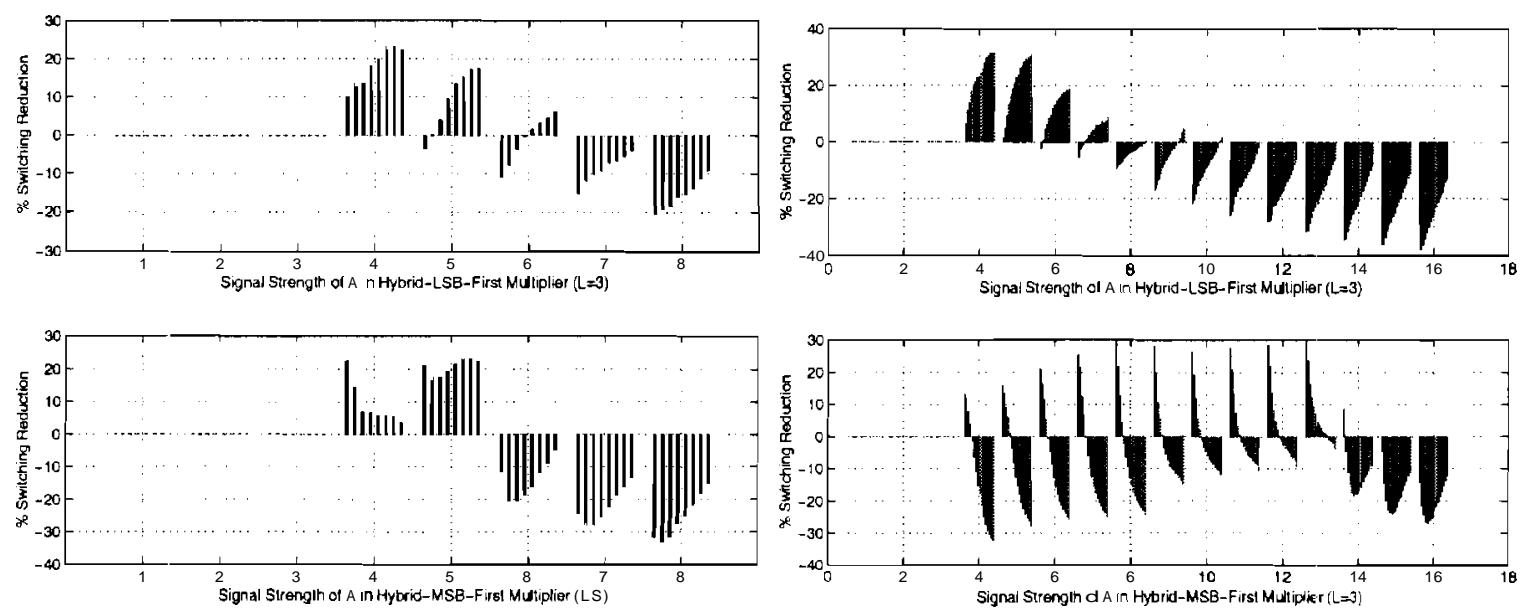

Fig. 11. $\eta_{\text {Tree }}$ for 8 (left) and 16-bit (right) hybrid array multipliers with $\mathrm{L}=3$ as a function of the signal strength in the operands.

of L. The reduction in switching activity under favorable signal conditions is as large as $40 \%$.

The relative performance of a large multiplier for small and large values of L is shown in figure 13 . This figures shows $\eta_{\text {Tree }}$ for 32-bit hybrid multipliers for $\mathrm{L}=1$ (figures on left) and $\mathrm{L}=8$ (figures on right), respectively. Since the indicated truncation for small signal strength completely annihilates its value, the first seven groups of bars are missing in the figures on right. No operations are necessary in this region of operation and no switching activity results in the hybrid multiplier, if such operands are applied. Switching reduction of up to $35 \%$ are achievable in the $\mathrm{L}=8$ case in comparison to about $30 \%$ for the $L=1$ case. Although the results shown in figures $8-13$, in general, suggest superior performance of hybrid LSB-first multiplier over hybrid MSB-first multipliers, one must remember that the decision to choose the best multiplication scheme is dependent on the input signal conditions. The relative switching 

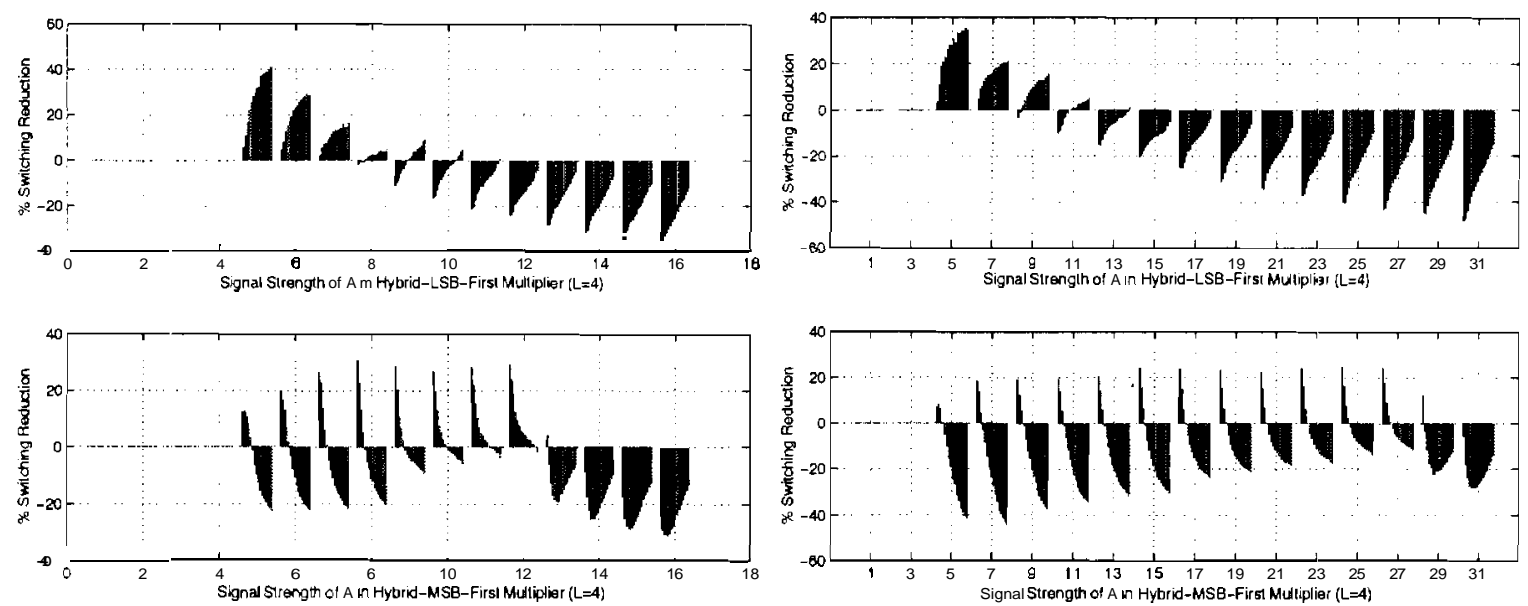

Fig. 12. $\eta_{\text {Tree }}$ for 16 (left) and 32-bit (right) hybrid array multipliers with L = 4 as a function of the signal strength in the operands.
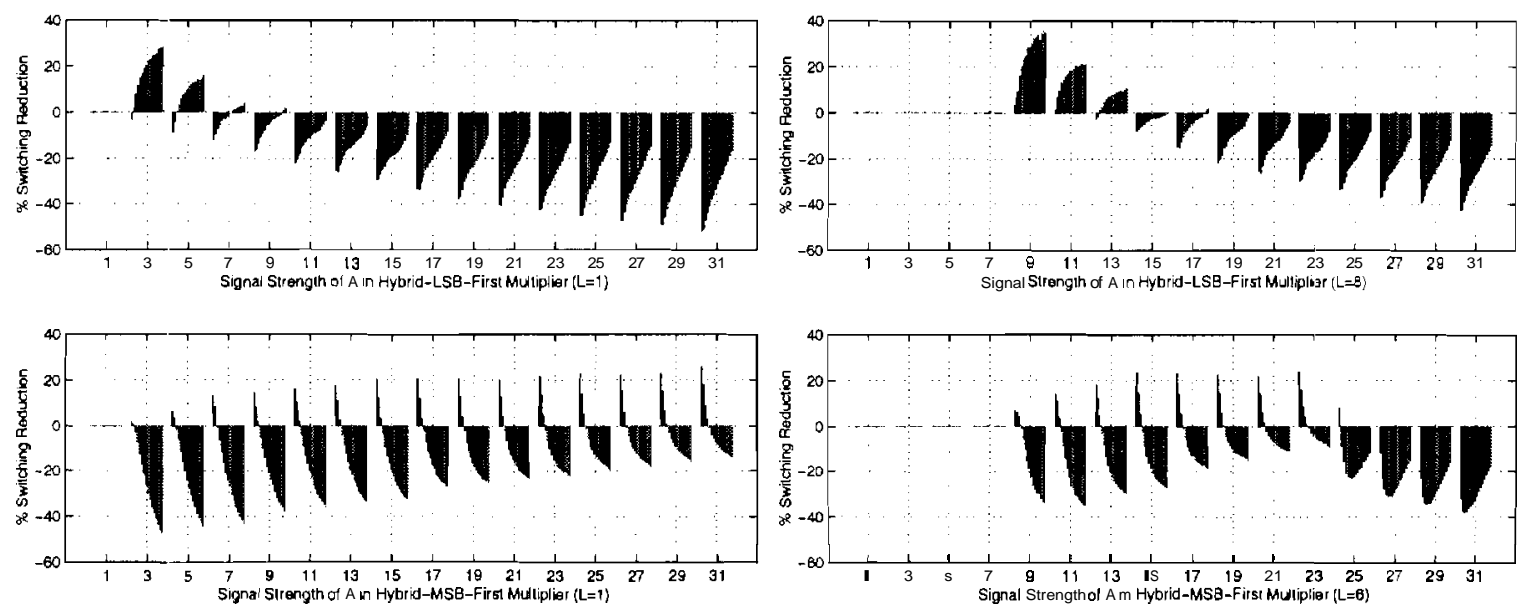

Fig. 13. $\eta_{\text {Tree }}$ for 32-bit hybrid array multipliers with $L=1$ (left) and $L=8$ (right) as a function of the signal strength in the operands.

activity reduction also depends on the choice of multiplier used in normalization in equation 1. Figure 14 demonstrates this point by showing $\eta_{\text {Array }}$ for 32-bit hybrid array multipliers with $\mathrm{L}=2$ and $\mathrm{L}=4$, respectively. Notice that the switching activity reduction in hybrid MSB-first multiplier, although smaller than its counterpart, is more consistent as signal strength of $\mathbf{A}$ varies. Hence, for a given application, the latter may be preferred despite its general inferior performance to the hybrid LSB-first multiplier.

B.2 Switchirg Activity for Correlated Signals

We now consider the performance of the presented multipliers using the $\mathcal{G}$ model. For this purpose we applied data samples obtained from Gaussian distribution for different signal strengths varying from 1 to $N-1$ bits. Four situations were chosen to reflect the effect of correlation in the signal by considering 

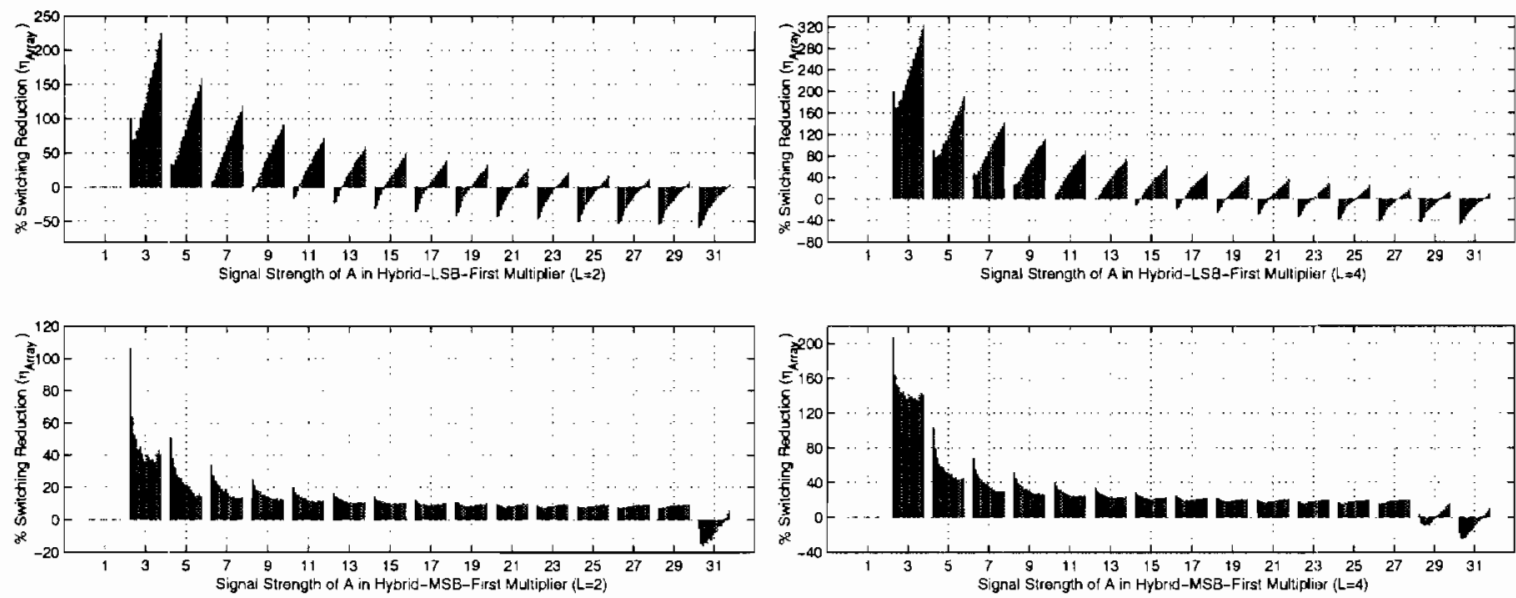

Fig. 14. $\eta_{\text {Array }}$ for 32-bit hybrid array multipliers with $L=2$ (left) and $L=4$ (right) as a function of the signal strength in the operands.


Fig. 15. $\eta_{\text {Tree }}$ for (left) 8-bit LSB-first CS, and, (right) 8-bit LSB-first RP, array multipliers as a function of the signal strength in. the operands. Values of $\rho_{A}$ and $\rho_{B}$ are shown with each plot.

high $(\rho=0.95)$ and low $(\rho=0)$ signal correlations. In figures $16-18$, results for $\rho_{A}=\rho_{B}=0$, $\rho_{A}=0, \rho_{B}=0.95, \rho_{A}=0.95, \rho_{B}=0$ and $\rho_{A}=\rho_{B}=0.95$ are shown in the figures on top left, top right, bottom left and bottom right, respectively. The correlated Gaussian signals were generated using an auto regressive $\mathrm{AR}(1)$ model [2], [3]. The results shown in each figure in this section were generated using 10,000 data vectors for each signal condition. Results are shown for only 8-bit multipliers as they are consistent for multipliers of all sizes. Figure 15 shows $\eta_{\text {Tree }}$ for LSB-first multipliers. In the case of LSB-first CS multiplier, correlation in B improves the reduction in switching activity, whereas, correlation in $\mathbf{A}$ reduces the gains. However, the change in $\eta_{T r e e}$ is nominal even for such high value of correlation. Higher value of $\rho_{B}$ causes a slight shift in the signal strength of $\mathbf{B}$ which causes maximum $\eta_{T r e e}$. The effect of increasing $\rho_{A}$ on LSB-first RP multiplier is to have slightly faster diminishing $\eta_{T \text { ree }}$ as signal 

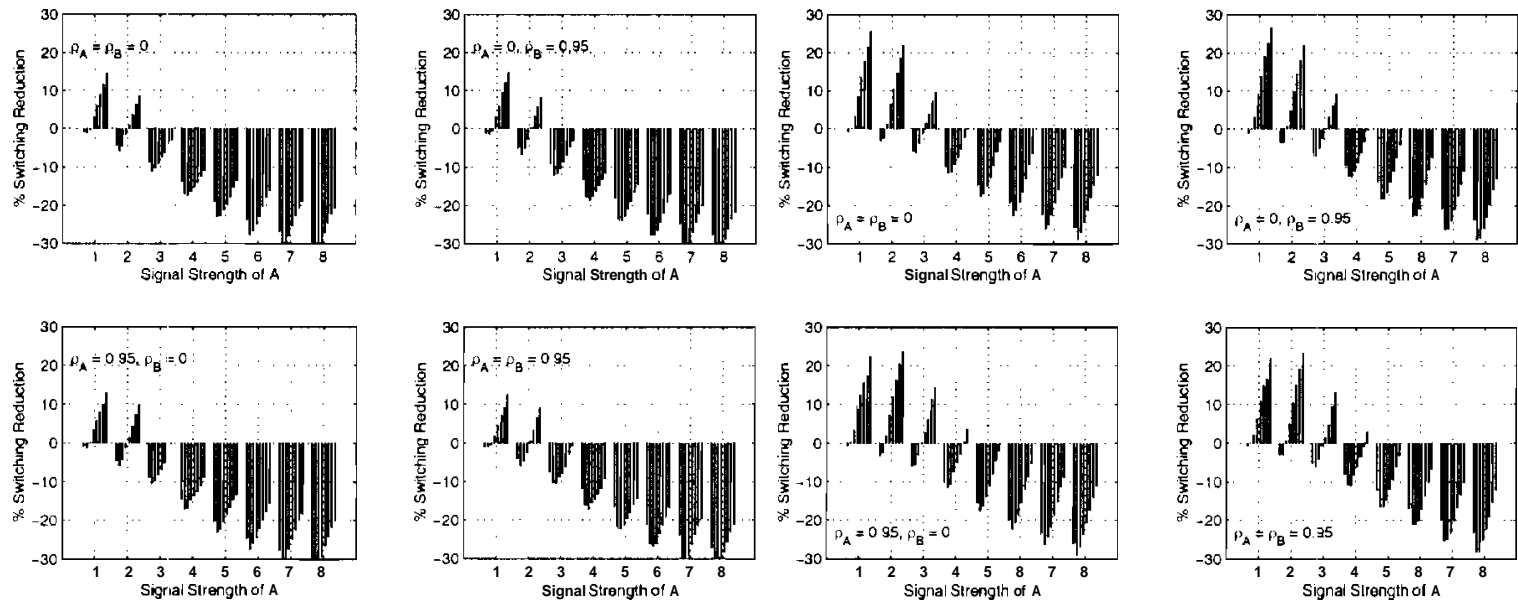

Fig. 16. $\eta_{\text {Tree }}$ for (left) 8-bit MSB-first CS, and, (right) 8-bit MSB-first RP, array multipliers as a function of the signal strength ir the operands. Values of $\rho_{A}$ and $\rho_{B}$ are shown with each plot.

strength of $\mathbf{A}$ increases. The effect of increasing $\rho_{B}$ is an "equalization" of $\eta_{\text {Tree }}$ for small signal strengths at A. However, the differences are very small.

In the case of MSB-first multipliers shown in figure 16, we notice that higher $\rho_{A}$ causes an "equalized" $\eta_{\text {Tree }}$ for small signal strengths of $\mathbf{A}$. Hence, better gains are obtained as signal strength of $\mathbf{A}$ increases, and these gains drop quickly as $\mathbf{A}$ becomes stronger. The effect of $\rho_{B}$ is not discernible Similar results are seen in the hybrid multipliers shown in figures $17-18$ which shows the effect of correlated signals on the performance of hybrid multipliers. In all these examples, the effect of $\rho_{B}$ is negligible, however, high $\rho_{A}$ causes the gains to equalize in the region where the hybrid multiplier out-performs the tree multiplier. It is noted that consideration of extremely high correlations do not make much sense because a better approach in this case is to difference the data and reduce its dynamic range. Hence, by adding overhead of add operation one can significantly reduce the size of the operands in multiplication. The results shown in this section clearly indicate that signal correlations have a small effect on the switching activity for all multipliers. It is actually the signal strength at the inputs which almost completely determines the switching in the multiplier. This is confirmed by a similar observation made for LSB-first RP multipliers in $[10]$

\section{B.3 Area Comparison}

The LSB-first CS and MSB-first CS multipliers were implemented in CMOS using $0.6 \mu$ technology. Both of these structures were implemented after inverter elimination simplifications for the partial product generator rows [4]. Cells were implemented for both non-inverted and inverted outputs [4] and the bottom most row constituted a vector merge adder for converting CS format to regular representation. The layout areas of the two multipliers is shown in table II for purpose of comparison. MSB-first CS adds a wiring overhead which results in an increased area. This is because the carry signal must be propagated one cell 

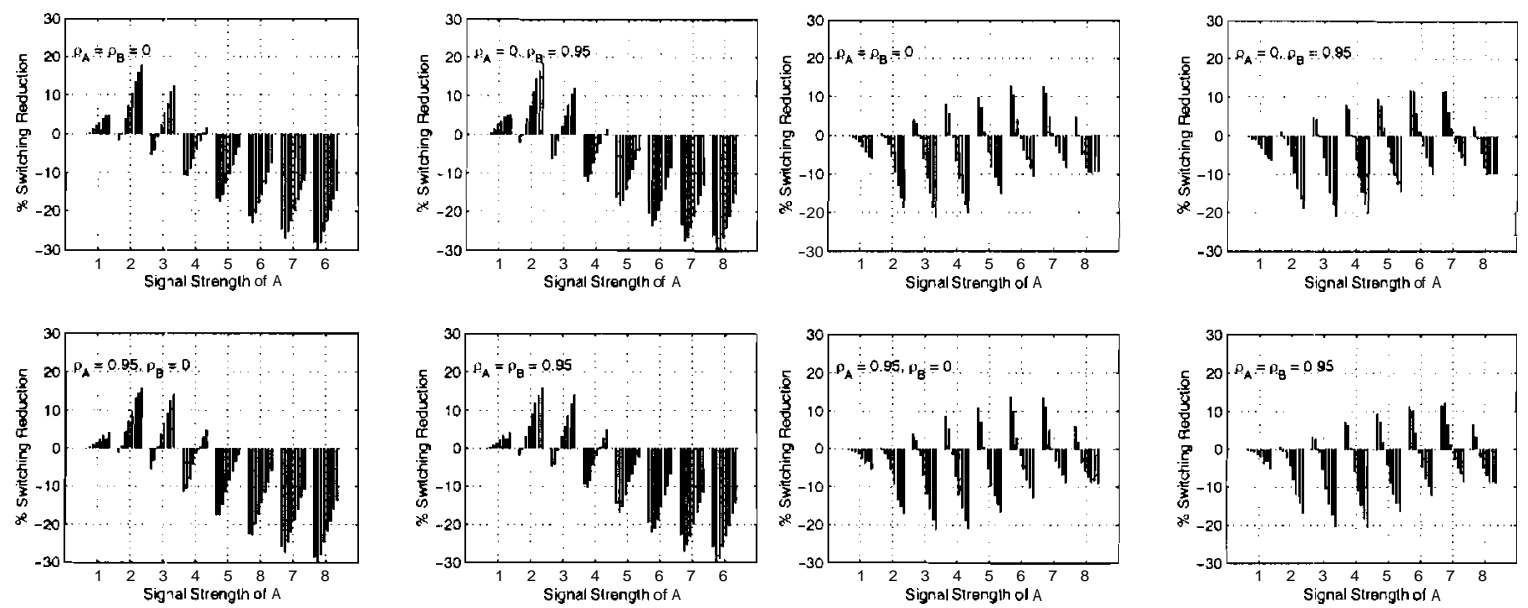

Fig. 17. $\eta_{\text {Tree }}$ for 8-bit hybrid array multipliers as a function of the signal strength in the operands. (left) Hybrid LSB-first CS/CS with $\mathrm{L}=1$, and, (right) Hybrid MSB-First RP/CS with $\mathrm{L}=1$. Values of $\rho_{A}$ and $\rho_{B}$ are shown with each plot.
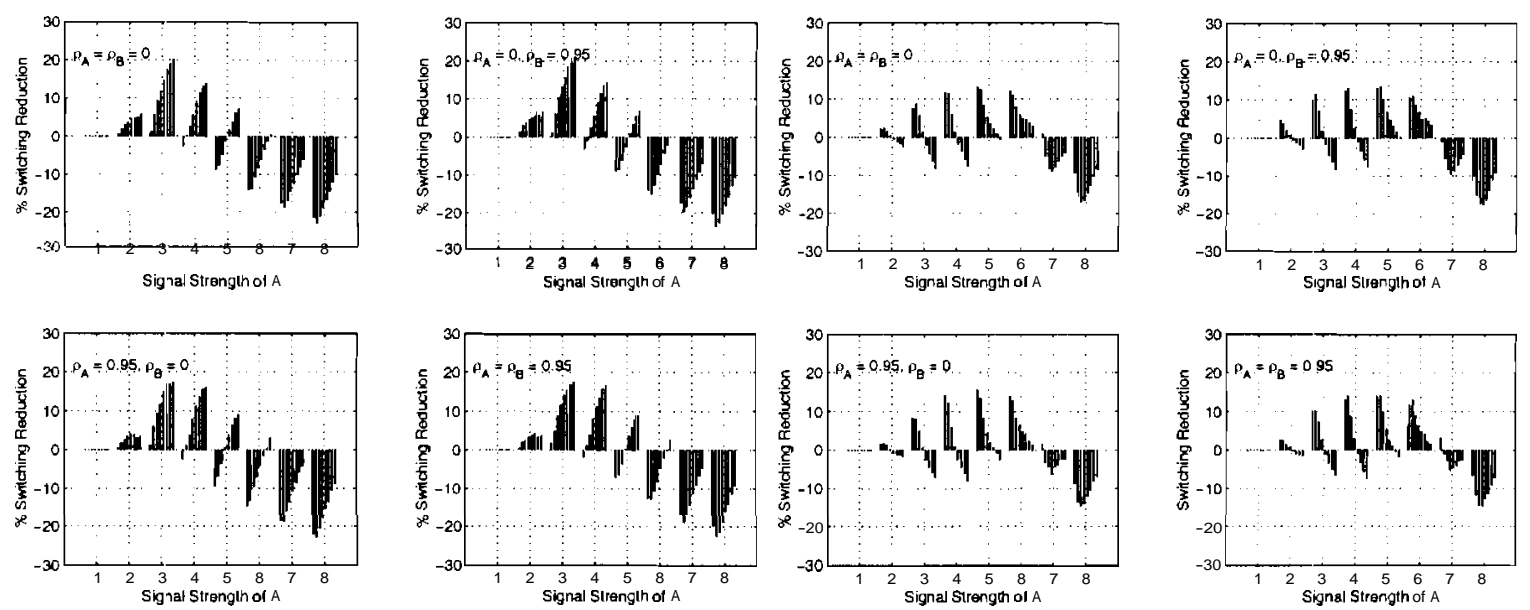

Fig. 18. $\eta_{\text {Tree }}$ for 8-bit hybrid array multipliers as a function of the signal strength in the operands. (left) Hybrid LSB-first CS/CS with $\mathrm{L}=2$, and, (right) Hybrid MSB-First RP/CS with $\mathrm{L}=2$. Values of $\rho_{A}$ and $\rho_{B}$ are shown with each plot.

further in a rectangular layout. These values can be used to approximately estimate the area overhead of using hybrid multipliers.

\section{Application to Low Power Design}

In the previous sections, we have provided a qualitative as well as quantitative assessment of the switching activity reduction which can be obtained by using the proposed multiplier structures for various signal conditions. These results can assist in the design for low-power as they show the relative strengths and weaknesses of different multiplier architectures. In this section, we will briefly discus:; the application of this work to low power quantization, reconfigurable computing and high-level synthesis for low-power 


\begin{tabular}{||c||c|c|c|c|c||}
\hline Multiplier & $N=8$ & $\mathrm{~N}=12$ & $\mathrm{~N}=16$ & $\mathrm{~N}=24$ & $\mathrm{~N}=32$ \\
\hline \hline LSB-first CS & 63,508 & 138,040 & 241,073 & 532,982 & 939,007 \\
\hline MSB-first CS & 84,948 & 178,406 & 306,009 & 663,954 & $1,158,672$ \\
\hline \hline Area Overhead & $33.8 \%$ & $29.3 \%$ & $26.9 \%$ & $24.6 \%$ & $23.3 \%$ \\
\hline
\end{tabular}

TABLE II

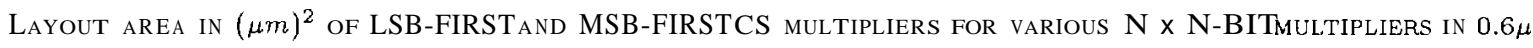
TECHNOLOGY

\section{A. Low Power Quantization}

As discussed earlier, non-dedicated DSP systems generally employ multipliers whose size is determined by the performance requirements of the most computationally expensive intended application. An application in a general DSP system with fixed resources may not require the full precision offered by the resource. In such a situation, the power dissipation of the computational unit can be significantly reduced by appropriate use of the resource. Such quantizations have been proposed in [8], [9] without considering support multiplier architectures. We further note that these results are also useful in formulating a strategy for employing variable word-length computing, in which different tasks of a DSP algorithm are computed with different precisions without significantly degrading the overall system performance.

As evident from the results presented in previous sections, the following two conditions must be met: first, an appropriate multiplier architecture should be selected, and second, correct input conditions must be provided such that reduced switching activity is guaranteed. Quite clearly, it is not enough to ensure only one of these conditions. For example, if we truncate the LSB bits of the B input in a LSB-first multiplier, it will not help reduce switching activity. Further, it is also important to ensure that favorable signal conditions are maintained at the inputs consistently. For example, if successive $\mathbf{A}$ inputs in the LSB-first multiplier have toggling $a_{0}$ bit, the reduction in switching activity will be entirely lost. Reduction in switching activity is possible only if the data-stream applied at $\mathbf{A}$ input of this multiplier ensures that successive sainples have all $L$ LSB bits turned off.

\section{B. Reconfigurable Computing}

The cellular array structure presented in section II is the most general template using which any array multiplier can be formed. In applications where reconfigurability is sought for the application at hand, one may use the c.nderlying structure proposed in this paper to form any of $\mathrm{N}$ ! possible multiplier architectures. It is noted that reconfigurability desired specifically for reduction of switching activity may not achieve that goal becsuse of the overheads involved. In general, these overheads reduce the speed of application as well as incresse the overhead power. However, for specific applications where structure of data stream is 
well-known, re-configurable multiplier may be employed which eliminates the undesired rows of multiplier (to form an appropriate hybrid multiplier) in order to increase the speed of multiplication. In such a case, the interpretation of array multipliers presented in section II and the template described in figure $1 \mathrm{can}$ prove to be extremely useful.

\section{Hzgh Level Synthesis Based on Knowledge of Signal Characteristics}

The results presented in this paper clearly indicate that each array multiplier offers advantages for specific signal conditions. Further, large improvements are possible in reduction of switching activity by appropriate choice of multipliers. Hence, maximum reduction in switching activity can be achieved by scheduling and allocating operations such that favorable input conditions are ensured at the inputs of the multipliers employed in the implementation. Hence, existing high-level synthesis tools can be improved such that they consider the expected signal behavior at various points of the algorithm while arriving at an implementation. Note that the condition of ensuring favorable signal conditions at the multiplier inputs also reduce bus-power, since these conditions must be met consistently between successive data samples. This work shows that an appropriate choice of array multiplier assures that reduction in switching activity in the input bus to the multiplier reflects as reduced switching activity in the multiplier Hence, one can reduce the pswer dissipation in a data-path by careful scheduling and allocation of instructions based on the expected statistical properties of the data being processed.

\section{Conclusion}

We presented several new array multiplier architectures for reducing switching activity in general digital signal processing applications. A general cellular structure was presented which can be used to obtain any array multiplier suitable for the given application. This structure provides a unified view of all $N$ ! possible N-bit array multipliers. The switching activity at the output nodes of the cells in various multiplier structures was analyzed and compared with a tree multiplier based on $4: 2$ cornpressors as well as a LSB-first CS array multiplier. It was shown that the relative improvement in power is a function of statistical properties of the input signals. It was also shown that selection of appropriate array architecture can give up to $40 \%$ reduction in switching activity compared to a tree multiplier, and more than $\mathbf{3}$ times reduction in switching activity compared to the widely used LSB-first array multiplier for commonly occurring situations. We also outlined applications of the proposed multipliers and the presented results to the areas of low power quantization, reconfigurable computing and high-level synthesis for low power. Hence, the proposed architectures can prove to be extremely useful structures for low power DSP system design.

\section{REFERENCES}

[1] E. E. Swartzlander, '.Computer Arithmetic," IEEE Computer Society Press, 1990.

[2] S. Haykin, "Adaptive Filter Theory," Prentice Hall, NJ, 1996. 
[3] J. G. Proakis and D. G. Manolakis, "Digital Signal Processing: Principles, Algorithms, and Applications," McMillan Publishing Company, New York, 1992.

[4] J. M. Rabzey, "Digital Integrated Circuits: A Design Perspective." Prentice Hall, New Jersey, 1996.

[5] N. H. E. Weste and K. Eshraghian, "Principles of CMOS VLSI Design: A Systems Perspective," 2nd Edition, Addison Wesley, 1994.

[6] S. E. McQuillan and J. V. McCanny, "A Systematic Methodology for the Design of High Performance Recursive Digital Filters," IEEE Trans. on Computers, Vol. 44, No. 8, pp. 971-982, Aug. 1995.

[7] J. K. Jain, L. Song and K.K. Parhi, "Efficient Semisystolic Architectures for Finite-Field Arithmetic," IEEE Trans. VLSI Systems, Vol. 6, No. 1, pp. 101-113, Mar. 1998.

[8] K. Muhammad and K. Roy, "On Complexity Reduction of FIR Digital Filters Using Constrained Least Squares Solution," In Proc. of 1997 IEEE International Conference on Computer Design (ICCD '97), pp. 196-201, Austin, Texas.

[9] K. Muhammad and K. Roy. "Low Power Digital Filters Based On Constrained Least Squares Solution," In Proc. of the 31 st Asilomar Conference on Signals, Systems and Computers, 1997, Monterey, California - Invited Paper.

[10] M. Lundberg, K. Muhammad, K. Roy and S. K. Wilson, "High-level Modeling of Switching Activity With Application to Low-power DSP System Synthesis," To appear in the 1999 Proc. IEEE International Conference On Acoustics, Speech, and Sig nal Processing (ICASSP'99).

[11] T. H. Cormen, C. E. Leiserson and R. L. Rivest, "Introduction to Algorithms," The MIT Press, 1990. 